\title{
Aspects of PLC-based in PID controller applied to control of oil pipeline located in Coari-Amazonas
}

\author{
Luiz H. C. Valda ${ }^{1}$, Marcello P. Ramos ${ }^{2}$, Nibson M. Calderon ${ }^{3}$, Antônio B. L. Medeiros ${ }^{4}$, Bruno J. Savino ${ }^{5}$, \\ Caio C. P. Cavalcante ${ }^{6}$, Henrique A. F. Menezes $^{7}$
}

1,2,3,4,5,6,7 Instituto de Tecnologia, Universidade Federal do Pará - UFPA - Belém - Pará - Brasil. Campus Guamá. Avenida Augusto Correa No. 01. CEP: 66075-110. Caixa Postal 479. PABX +55 9132017000.

E-mail: luiz_valda@yahoo.com.br,mar.pramos@gmail.com,nibjr@hotmail.com, benjaminleao@gmail.com, bruno.judiss@gmail.com, caiocpcavalcante@gmail.com, henriqueadativafmenezes@gmail.com.

Received: December 17th, 2016

Accepted: February $23^{\text {th }}, 2017$

Published: March 30 3017

Copyright $@ 2016$ by authors and Institute of Technology Galileo of Amazon (ITEGAM) This work is licensed under the Creative Commons Attribution International License (CC BY 4.0).

http://creativecommons.org/licenses/by/4.0/ cc) (i) (3) Open Acees

\begin{abstract}
One of the main sources of petroleum products beneath ground in Brazil it is obtained from the Amazon forest. Where the main problem faced during the flow of this product is to maintain the constant pressure and flow at the highest possible level. In this work, the parameters such as the pressure and flow of a pipeline are constantly maintained through the implementation of control valves, depending on the difference of pressure, flow and temperature in the pipeline. PLC (Programmable Logic Controller) is used to automatically regulate the flow and pressure during transportation of the oil by controlling the percentage of opening of the control valves. The desired setpoint for pressure and flow is obtained by implementing a suitable controller that regulates the operating variables of the piping. For this, a PLC-based PID controller is developed and it is openloop responses are identified. Simulation studies are performed on the MATLAB/SIMULINK platform to ensure the performance of the controller. The Controller Regulation is done by ZN (Ziegler and Nicholas) PID and the results prove that it provides consistent performance relative to the unit's operational requirements.
\end{abstract}

Keywords: PID Controller, pipeline, pressure.

\section{Aspectos de controle PID baseado em CLP Aplicado ao controle de duto petrolífero localizado em Coari-Amazonas}

\section{RESUMO}

Uma das principais fontes de produtos petrolíferos em terra no Brasil é obtido a partir da floresta amazônica. Aqui, o principal problema enfrentado durante o escoamento deste produto é manter a pressão constante e vazão no maior patamar possível. Neste trabalho, os parâmetros tais como a pressão e vazão de um oleoduto são mantidos constantemente através da implementação de válvulas de controle, dependendo da diferença de pressão, vazão e temperatura no duto. CLP (Controlador Lógico Programável) é usado para regular automaticamente a vazão e a pressão durante o transporte do petróleo através do controle da porcentagem de abertura das válvulas de controle. O ponto de ajuste desejável para a pressão e vazão são obtidos através da implementação de um controlador adequado que regula as variáveis operacionais da tubulação. Para isso, um controlador PID baseado em PLC é desenvolvido e suas respostas em malha aberta são identificadas. Os estudos de simulação são realizados na plataforma MATLAB/SIMULINK para garantir o desempenho do controlador. A Regulação do controlador é feito por ZN (Ziegler e Nicholas) PID e os resultados provam que proporciona um desempenho consistente com relação aos requisitos operacionais da unidade.

Palavras Chaves: Controle PID, Oleoduto, Pressão.

\section{INTRODUÇÃO}

Petróleo (literalmente, "óleo de rocha") é uma substância que se formou sob a superfície da terra ao longo dos anos. Os restos de plantas e animais antigos foram enterrados e comprimidos sob milhares de metros de areia, lama e rocha. Os materiais orgânicos, em certas condições geológicas, foram transformados por pressão de 
sobrecarga e calor subterrâneo em compostos hidrocarbonetos, oleodutos entre locais de produção, refinarias e pontos de consumo. como petróleo bruto e gás natural. Grandes quantidades de petróleo Durante muitos anos, os embarques de petróleo representaram cerca bruto e produtos refinados de petróleo são transportados por navio e de $30 \%$ do comércio marítimo mundial.

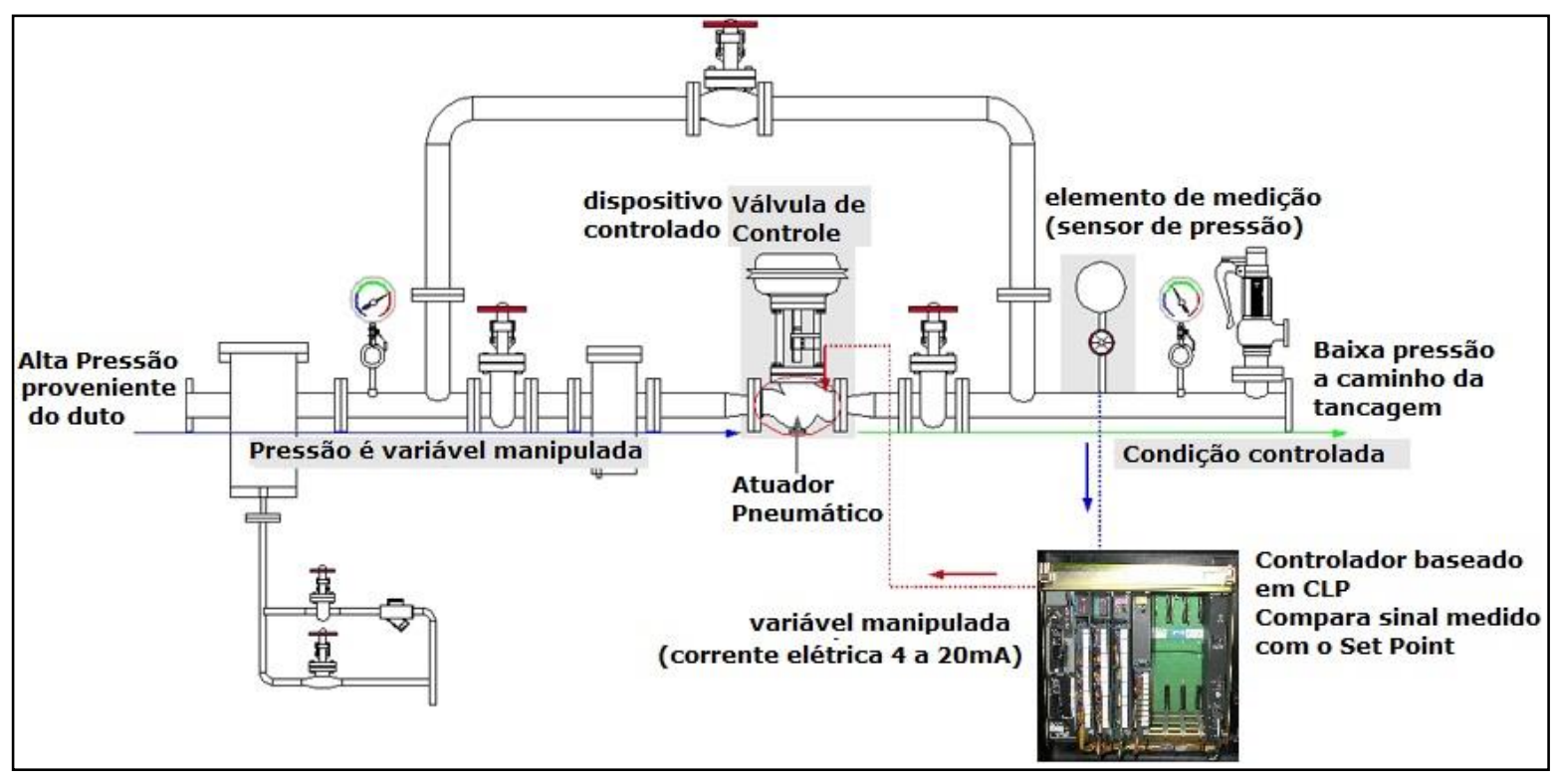

Figura 1: Layout do tramo de controle de pressão.

Há muitos procedimentos envolvidos no transporte do petróleo ou dos derivados de petróleo em alto mar para a região costeira e rios navegáveis e alcançado de forma eficaz através do transporte duto viário. Os oleodutos são selecionados com base em vários critérios, tais como, ciclo de vida, exposição contínua em sal ou outros elementos abrasivos, água do mar e raios solares, estratégias econômicas e implantação dos acessórios em todo o sistema de transporte. Para o transporte, o oleoduto utilizado é feito de materiais a base de aço-carbono por causa da alta pressão e do parâmetro tolerância de fluxo [1][2].

De acordo com a Conferência das Nações Unidas sobre Comércio e Desenvolvimento (UNCTAD), os embarques de petróleo bruto totalizaram 1,78 bilhão de toneladas em 2012, enquanto os embarques de produtos refinados e de gás liquefeito totalizaram 1,05 bilhão de toneladas (aproximadamente 230 milhões de toneladas de GNL). No transporte em longas distancias, existem vários problemas identificados pelos agentes logísticos com base em pressões constantes de pressão e fluxo [3][4].

Atualmente, existe uma malha linear especial implantada com sensores em especial nas cabeças dos dutos para monitorar e regular o fluxo e a pressão com que estes interagem com os terminais de transferência e estocagem. Eles demonstraram sistemas de comunicação multicamadas que asseguram o encaminhamento eficaz de dados entre os sensores, mas não há consideração de válvula de controle entre as redes de comunicação remota baseadas em sensores que envolve operação manual para alcançar o desempenho desejado do transporte de oleodutos [5].

Para superar as dificuldades práticas, este trabalho apresenta a implementação das válvulas de controle automáticas para as faixas de pressão e fluxo desejadas [6].

\section{MATERIAIS E MÉTODOS}

O modelo esquemático do sistema industrial em análise é mostrado na Figura 1. A instalação inclui sensor de pressão, válvula de controle e o CLP. A válvula de controle é implementada ao final do duto, imediatamente antes do terminal de estocagem. Para fins de controle, a configuração foi interconectada com o CLP Allen Bradley PLC-5, que é programado com controlador PID. A Figura 2 mostra o diagrama de fluxo do processo do sistema de transporte de óleo.

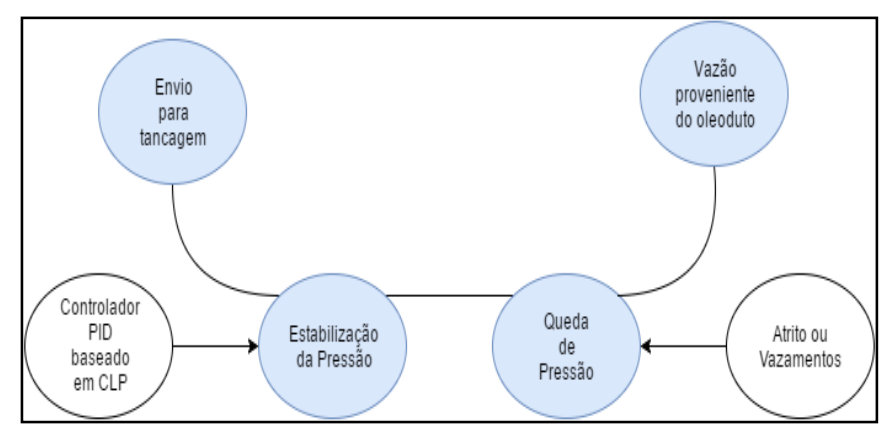

Figura 2: Fluxograma do processo.

\section{ESPECIFICAÇÕES DO MODELO}

Para a modelagem da planta de processo, a resposta em malha aberta é obtida pelo levantamento histórico do comportamento dinâmico da planta e realização de testes experimentais em simulação computacional. A vazão de petróleo é obtida ajustando a através de medidores ultrassônicos de vazão ao longo do duto. 
A abertura e fechamento da válvula de controle é controlada usando um atuador pneumático. Para isso, a resposta em circuito aberto é realizada regulando o fluxo de petróleo, ajustando a abertura e fechamento da válvula conforme apresenta Tabela 1.

Tabela 1: Parâmetros identificados do modelo.

\begin{tabular}{c|l|l|l|l|l|l|l}
\hline \multicolumn{2}{l}{$\begin{array}{l}\text { Percentual } \\
\text { de vazão }\end{array}$} & & Kp & & Tp (s) & & $\theta(\mathrm{s})$ \\
\hline 20 & & & 0.8520 & & 24.297 & & 5.835 \\
\hline 40 & & & 0.5799 & & 39.69 & & 3.31 \\
\hline 60 & & & 0.3777 & & 6.153 & & 1.347 \\
\hline 80 & & & 0.1871 & & 32.968 & & 2.032 \\
\hline
\end{tabular}

A indicação em negrito denota o pior caso do modelo para o ganho de processo máximo juntamente com a menor constante de tempo e tempo de atraso [8].

A partir da Tabela 1, é claro que o comportamento do processo é não-linear e estável. Assim, a função de transferência $(G(s)=$ $\left.\left[\frac{K_{p}}{\tau_{p} S+1}\right] e^{-\theta s}\right)$ é usada para representar a manutenção da vazão de petróleo. O pior caso com o maior ganho de processo e a menor constante de tempo é selecionado para exemplificar o modelo de processo. O modelo identificado do processo de controlo de vazão é representado como,

$$
(s)=\frac{0.8520}{6.153 S+1} e^{-1.347 s}
$$

Onde, $\mathrm{Kp}=$ ganho do processo $(0.8520), \tau \mathrm{p}=$ constante de tempo (6.153) e $\theta=$ atraso do processo (1.347) obtido a partir dos dados compilados.

\section{III.1 CONCEPÇÃO DO CONTROLADOR}

Muitos pesquisadores anteriores usaram o desempenho de um controlador convencional como ponto de referência para analisar o desempenho de um controlador proposto. Desta forma, o controlador convencional, tal como o controlador PID de ZieglerNicholas e o controlador PID baseado no Controlo de Modelo Interno IMC-PID [7][8] são considerados para fins comparativos e os parâmetros do controlador são dados como,

$$
\begin{gathered}
K_{c}=\frac{\mathrm{a} \tau_{p}}{\theta K_{p}} ; \quad a \in[1.2,2] ; \tau_{i}=2 \theta ; \\
\tau_{d}=0.5 \theta[\mathrm{ZN}-\mathrm{PID}] \\
K c=\frac{\tau p+0.5 \theta}{K p(\lambda+0.5 \theta)} ; \quad \tau \mathrm{i}=\tau \mathrm{p}+0.5 \theta ; \\
\tau \mathrm{d}=\frac{\tau \mathrm{p} \theta}{2 \tau \mathrm{p}+\theta}[\text { Simple }- \text { IMC PID }] ;
\end{gathered}
$$

Onde Kc é o ganho do controlador, $\tau$ i é a integral de tempo, $\tau$ d é o tempo de derivação e $\lambda$ é a constante de tempo em loop fechado.
Tabela 2: Parâmetros do controlador.

\begin{tabular}{l|l|l|l|l|l|l}
\hline \multicolumn{2}{l|}{ Controladores } & \multicolumn{4}{l|}{ Parâmetros } & \\
\hline & & $\mathrm{Kc}$ & $\mathrm{Ti}(\mathrm{s})$ & $\mathrm{Td}(\mathrm{s})$ & $\begin{array}{c}\mathrm{Ki}= \\
(\mathrm{Kc} / \mathrm{Ti})\end{array}$ & $\begin{array}{c}\mathrm{Kd}= \\
(\mathrm{Kc} * \mathrm{Td})\end{array}$ \\
\hline & & & & & & \\
\hline ZN-PID & 6.4339 & 2.694 & 0.6735 & 2.4288 & 4.3332 \\
\hline IMC-PID & 10.557 & 1.5255 & 0.37549 & 6.992 & 3.9631 \\
\hline \multicolumn{2}{l|}{ Shams-IMC-PID } & 2.508 & 4.558 & 2 & 0.5466 & 4.508 \\
\hline
\end{tabular}

O ajuste Shams-IMC é um método de ajuste de loop fechado que supera a deficiência do conhecido método de ciclagem contínua Ziegler-Nicholas e dá consistentemente melhor desempenho e robustez para o processo de primeira ordem. Utilizando esta afinação, é possível obter o desempenho de rejeição de perturbações melhorado ajustando o parâmetro de sintonização único do controlador. Os parâmetros do controlador Shams IMCPID [9] são dados por,

$$
K c=\frac{\alpha}{\operatorname{Kp}(2 \tau i-\alpha+\theta)} ; \tau i=\alpha
$$

Onde Kc é o ganho do controlador e $\tau i$ é o tempo integral. $\mathrm{O}$ valor de $\alpha$ (elemento de fase não-mínima) é selecionado de modo que cancela o pólo em $\mathrm{s}=-1 / \tau$ e o valor de $\alpha$ é obtido como;

$$
\alpha=\tau\left\{1-\left(1-\frac{\tau c}{\tau}\right)^{2}\right\} e^{-\theta \tau} ; \quad \tau d=\tau 2 ;
$$

$\tau c=2 \theta$

A característica importante deste controlador é que ele lida com processos não-lineares e estáveis de forma unificada. A partir das regras de ajuste, $\tau c$ controla o tradeoff entre robustez e desempenho do sistema de controle. As configurações dos referidos controladores acima são calculadas com base no modelo FOPTD fornecido e os parâmetros do controlador são fornecidos na Tabela 2 .

\section{III.2 INTERFACEANDO O SISTEMA EM TEMPO REAL COM SCADA}

Um modelo SCADA já em utilização em um processo industrial real é adaptado desenvolvido para monitorar todo o processo e para visualizar / variar os valores de sintonia dos controladores PID ZN, IMC e Sham-PID sintonizada remotamente. A tela desenvolvida no SCADA usando o software GE IFIX, uma visão da tela é mostrada na Figura 3. Aqui a faixa de vazão e pressão é ajustada para a qual o atuador da válvula é acionado, correspondentemente a pressão para o mesmo resultado de vazão é monitorada e a abertura da válvula de controle necessária é feita pelo controlador automaticamente com base nos sinais de controle. Todos os parâmetros da instalação experimental são monitorados e controlados através do SCADA. O painel de controle do SCADA consiste na visualização numérica das leituras de pressão lidas pelo sensor de pressão. Também mostra o percentual de abertura da válvula de controle.

A luz do alarme pisca quando a pressão do petróleo ou a vazão do petróleo ultrapassa o setpoint de segurança. O botão de emergência no painel de controle é usado para fechar todo o sistema em caso de qualquer emergência [10][11]. 
Valda et al., ITEGAM-JETIA. Vol. 03, № 09, pp.73-78. Março, 2017.

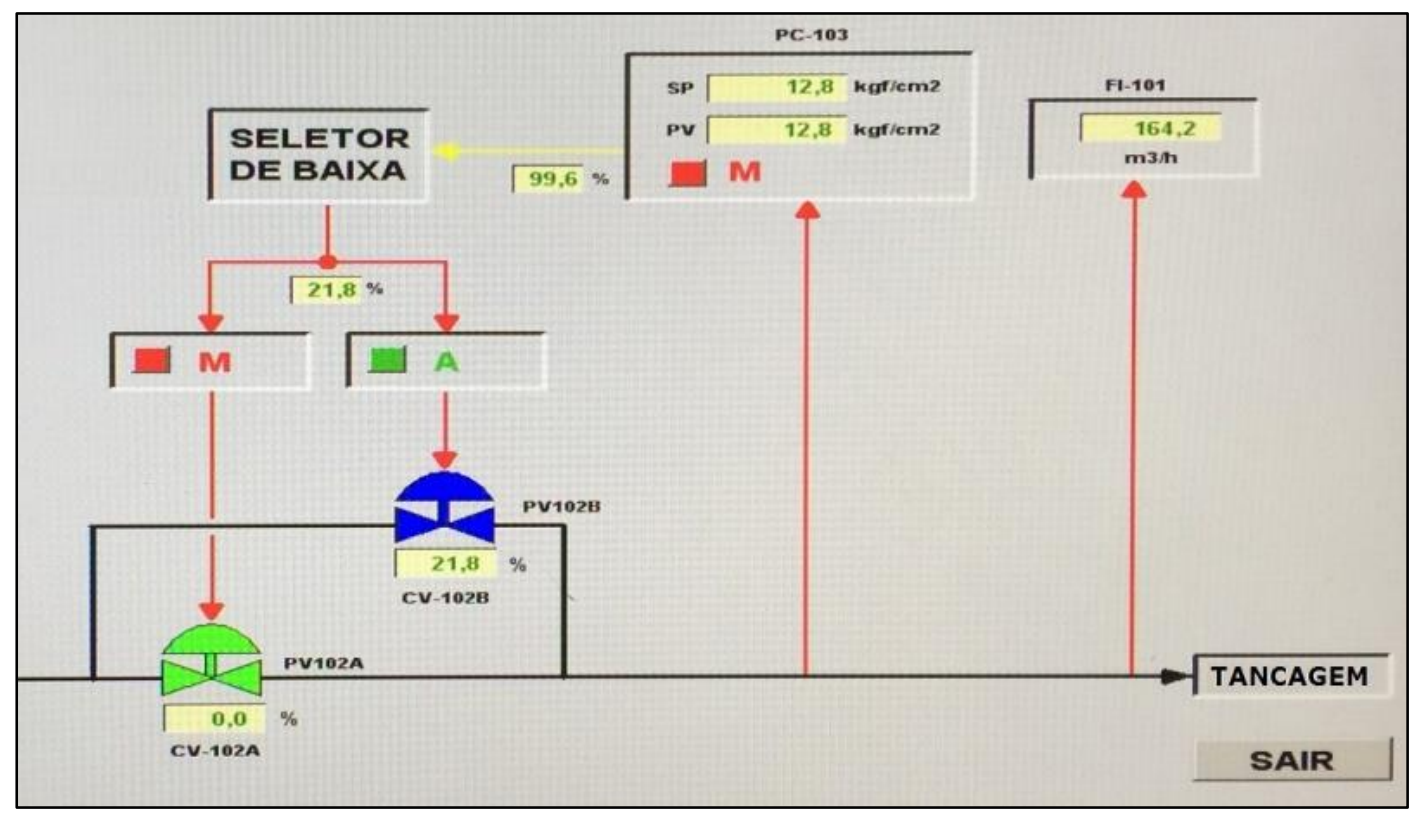

Figura 3: Tela do SCADA para manter constante a vazão e pressão de petróleo através do oleoduto.

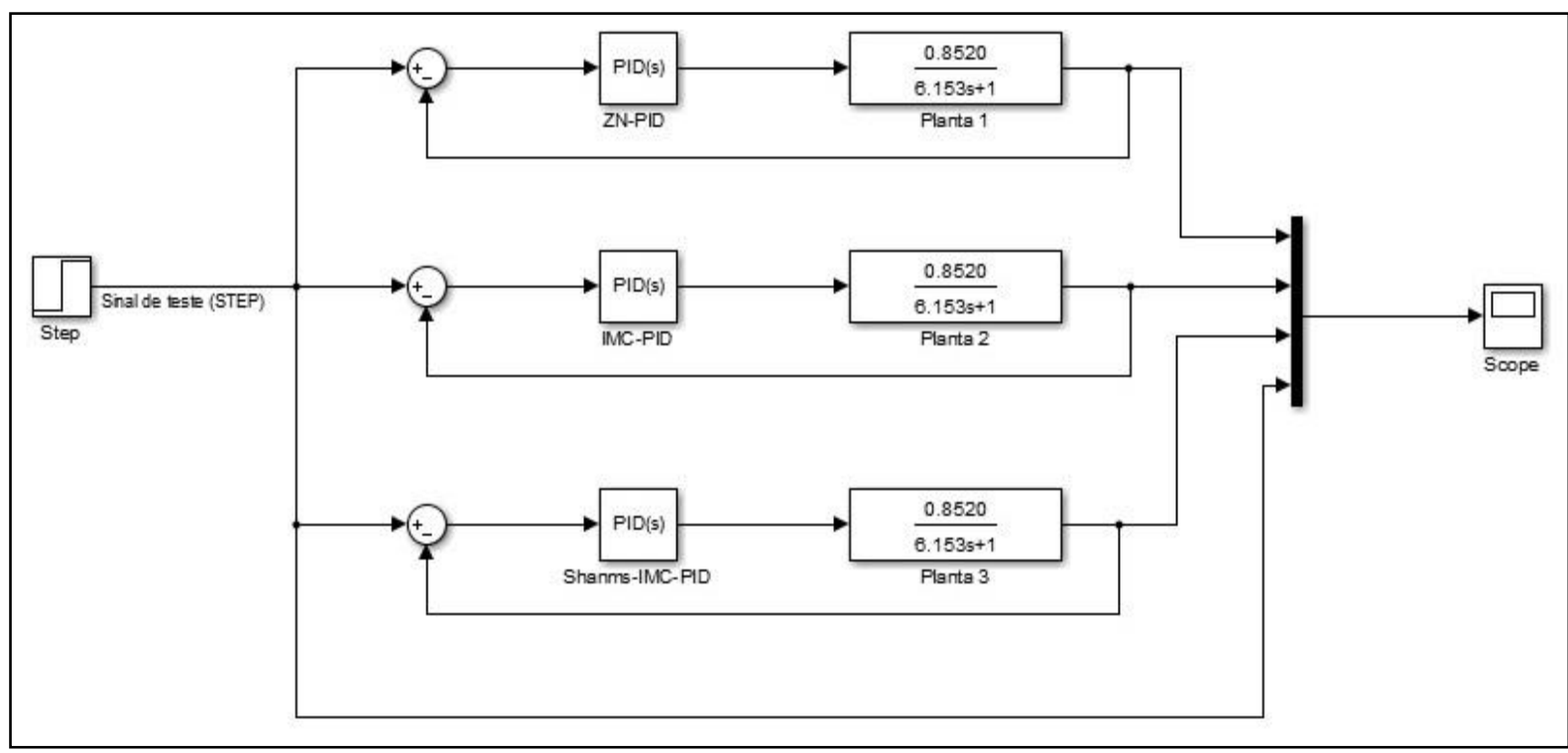

Figura 4: Modelo no MATLAB/Simulink.

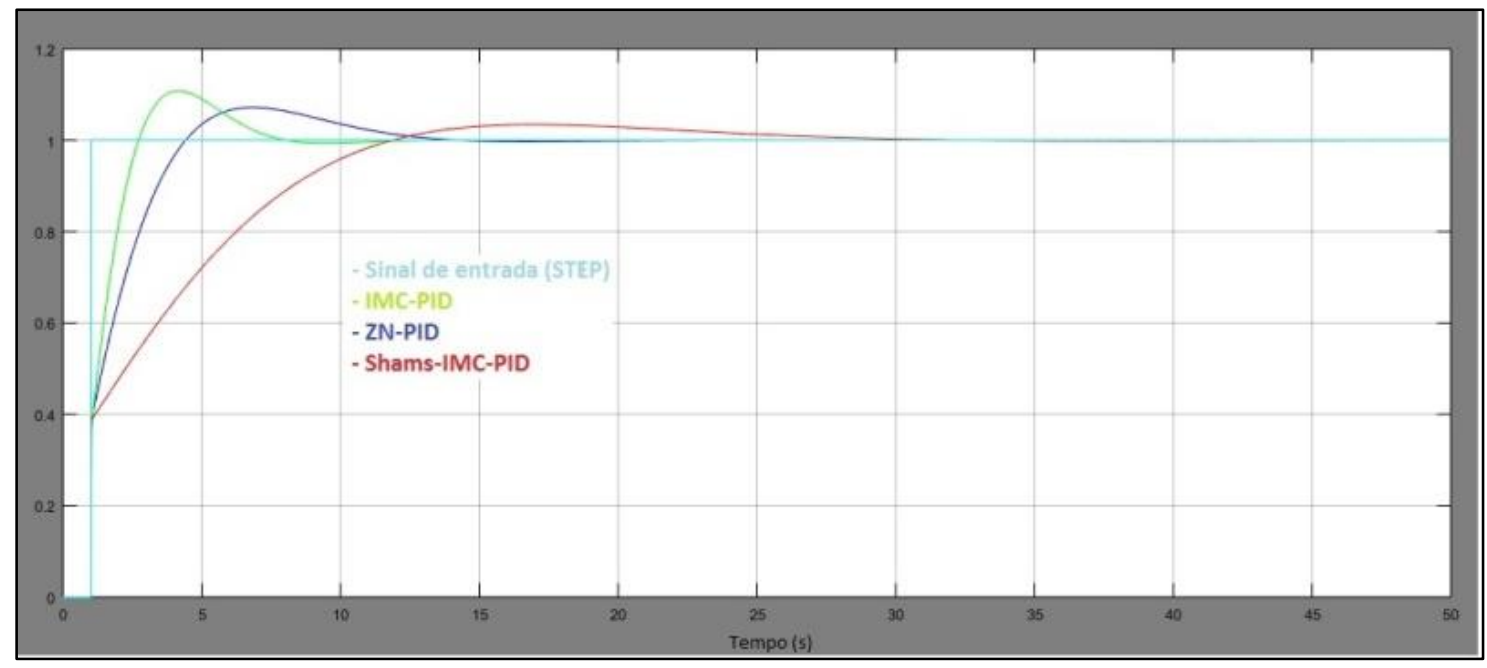

Figura 5: Análise de performance usando modelo no MATLAB/Simulink. 


\section{III.3 ANÁLISE DE DESEMPENHO}

No estudo de simulação, os desempenhos de vários controladores são comparados estabelecendo o pico do valor máximo de incerteza (Ms) para comparação. É melhor ter IAE, TV e Ms (Absoluto de Integração, Variação Total da entrada, valor de margem de incerteza) para ser pequeno, mas para um controlador bem ajustado há um trade-off, o que significa que uma redução no IAE implica um aumento na TV e Ms (e vice-versa) [12].

A partir da análise de desempenho os resultados da simulação revelam que o controlador IMC-PID do Shams é forçado a seguir o set point em curta duração de tempo e para manter o estado estacionário sem overshoot e com índices mínimos de erro e índices de boa qualidade em comparação com $\mathrm{ZN}$ e controlador simples IMC-PID [13]. Também proporciona uma rejeição de perturbações mais rápida com o tempo de duração de 8s e uma sobrecarga menor de 22,57\% em comparação com controladores ZN e Simple IMC-PID que rejeitam a mesma perturbação com o período de 11 e 18 s com overshoot de $39,24 \%$ e $26,12 \%$, respectivamente, mantendo o ponto de ajuste.

\section{III.4 TESTE DE REJEIÇÃO DE PERTURBAÇÕES}

O desempenho da rejeição de perturbações é investigado no ponto de operação de baixa taxa a $150 \mathrm{~m}^{3} / \mathrm{h}$. Uma perturbação de passo é introduzida no processo por meio do aumento da taxa vazão para $180 \mathrm{~m} / \mathrm{h}$ após o período de tempo de 50 s e é assegurado que apenas o controlador Shams IMC-PID amorteça a perturbação num período de tempo mais curto de $21 \mathrm{~s}$ e com menor undershoot de 14,28\% em comparação com os controladores ZN-PID e Simple-IMC PID. Os índices de erro e de qualidade do sinal de saída são utilizados para avaliar o desempenho de rejeição de perturbações dos controladores.

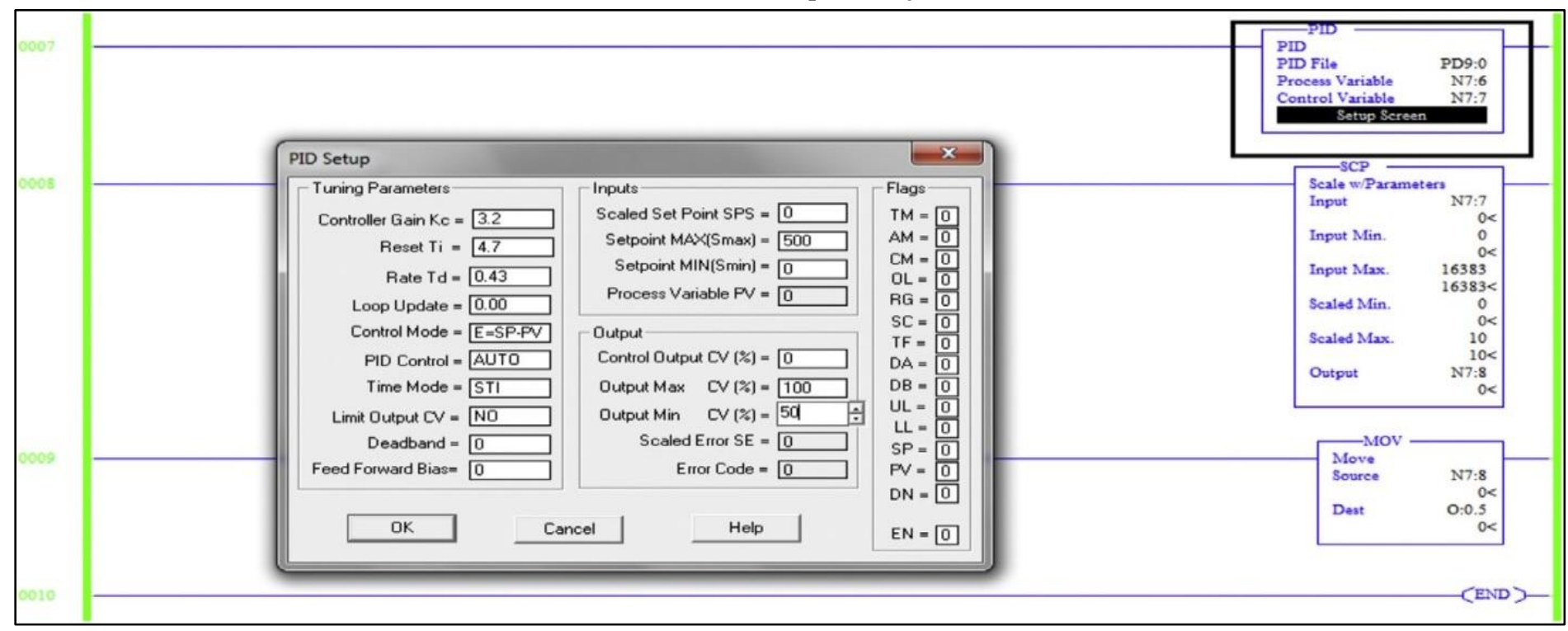

Figura 6: Bloco de controle PID no qual os valores de sintonização foram introduzidos para criar uma malha de sistema fechada, dando feedback à válvula de controle.

\section{RESULTADOS EXPERIMENTAIS}

É criado um bloco PID baseado no programa da lógica em linguagem Ladder no CLP através do qual os valores de Kc, Ki, Kd são introduzidos no bloco PID que é mostrado na Figura 6. As respostas do Ponto de Ajuste do Passo correspondente com vários processos de overshoots de primeira ordem mais tempo de atraso, $\mathrm{G}(\mathrm{s})=0,85 /(6,15 \mathrm{~s}+1)$ e-1,34s por alteração do valor de Kc. Uma experimentação em tempo real é realizada através de simulação computacional para analisar o desempenho dos controladores Shams-IMC-PID ZN e IMC simples no sistema de circuito fechado com uma faixa de operação de $180 \mathrm{~m}^{3} / \mathrm{h}$ com duração de tempo de $\mathrm{t}=0-90 \mathrm{~s}$ e os índices de desempenho correspondentes também são calculados.

A partir dos resultados em tempo real, fica claro que a resposta do controlador Shams-IMC-PID atinge o setpoint em 23s e mantém o estado estacionário para todo o período de medição de até $\mathrm{t}=90 \mathrm{~s}$. Por outro lado, o controlador ZN-PID atinge o ponto de ajuste em 46s e mantém-se com ligeira oscilação até $\mathrm{t}=60 \mathrm{~s}$. Um controlador IMC-PID simples atinge o ponto de ajuste em 29 s, mas leva a oscilação mínima ao longo do setpoint até atingir a duração total de 90s. O controlador Shams-PID fornece índices de erro mínimos de 16,54\% em comparação com controladores convencionais e proporciona o melhor desempenho para a regulação da vazão e pressão no transporte de oleoduto.

\section{CONCLUSÃO}

Neste trabalho, a manutenção da taxa de pressão constante é obtida quando o petróleo é transmitido através da cabeça do oleoduto, imediatamente a montante do terminal de transferência e estocagem petrolífero. Um controlador PID baseado em CLP é desenvolvido juntamente com o SCADA para o monitoramento e controle on-line e para a sintonia de controlador PID com base em CLP, controladores ZN, IMC simples e Shams IMC-PID são analisados. Uma análise computacional baseada em simulação é realizada em termos de resposta do sistema é apresentada. A simulação e os resultados experimentais comprovam que o controlador Shams IMC-PID proporciona um comportamento transitório e estável, com índices mínimos de erro e índices de boa qualidade em comparação com os controladores simples IMC-PID e ZN. Entre as regras de ajuste do controlador, o controlador Shams 
IMC-PID fornece 34\% melhor desempenho e também tolerou as perturbações nos parâmetros do modelo quando o setpoint muda.

Além disso, produz um desempenho bem sucedido no teste de rejeição de perturbações. Finalmente, o problema de variar manualmente a abertura da válvula de controle para atingir a pressão desejada é substituído por um controlador PID IMC-PID com base no CLP.

\section{REFERÊNCIAS}

[1]. HOU, T.;LIU, W.L.;LI, L. Intelligent remote monitoring and diagnosis of manufacturing processes using an integrated approach of neural networks and rough sets. Journal of Intelligent Manufacturing. 2003. 14(2), p. 239-253.

[2]. ZHANG， L.B.;WANG，Z.H.;LIANG，W. Remote and intelligent diagnostic methods on leakage fault for crude oil pipelines. In 2006 international pipeline conference. American Society of Mechanical Engineers. 2006. p.683-688.

[3]. HU, W.; STARR, A.G.; ZHOU, Z.; LEUNG, A.Y.T. A systematic approach to integrated fault diagnosis of flexible manufacturing systems. International Journal of Machine Tools \& Manufacture. 2000. 40(11), p. 1587-1602.

[4]. VENKATASUBRAMANIAN, v.; RENGASWAMY, R.; YIN, K.; KAVURI, S.N. A review of process fault detection and diagnosis part 1: Quantitative model-based methods. Computers and Chemical Engineering. 2003. 27(3), p. 293-311. 